\title{
Cardiology
}

\section{sST2 Protein Serum Levels in Patients with Chronic Heart Failure}

\author{
R. E. Tokmachev, PhD; A. Ya. Kravchenko, PhD, ScD; A. V. Budnevsky, PhD, ScD; \\ E. S. Ovsyannikov, PhD*; T. A. Chernik; E. V. Tokmachev, PhD; Ya. S. Shkatova \\ Voronezh State Medical University named after N.N. Burdenko \\ Voronezh, the Russian Federation
}

\begin{abstract}
The purpose of this study was to determine the blood concentration of SST2 and evaluate the correlations between sST2 and the clinical, instrumental, and echocardiographic characteristics of patients with $\mathrm{CHFrEF}$ (chronic heart failure with reduced ejection fraction) and CHFpEF (chronic heart failure with preserved ejection fraction), as well as to evaluate the sST2 informativeness in comparison with NT-proBNP.

The study involved 160 patients diagnosed with CHF ( 86 men and 74 women, mean age of $72.4 \pm 8.7$ years). According to the LVEF value, the patients were divided into two groups: $\mathrm{CHFpEF}(\mathrm{EF} \geq 50 \%)$ was recorded in 69 people, $\mathrm{CHFrEF}(\mathrm{EF}<50 \%)$ - in 91 people. The functional class (FC) of CHF was determined according to the NYHA classification (1994). NT-proBNP and hsCRP levels had a stronger positive association with sST2 levels in CHFrEF patients than in CHFpEF patients. The results obtained (regardless of the EF) indicated a statistically significant direct correlation between the levels of sST2 and LAVI. The weak direct correlation between SST2 and NYHA FC, in contrast to the direct strong correlation between NT-proBNP and sST2, indicates that sST2, which is quite important both for determining the current state of the patient and for predicting the course of the disease, still cannot be used as a single and universal indicator. In this regard, further comprehensive study of the diagnostic and prognostic capabilities of the sST2 and NT-proBNP biomarkers in CHF patients with different EF is required. (International Journal of Biomedicine. 2020;10(4):342-346.)
\end{abstract}

Key Words: sST2 $\bullet$ NT-proBNP • hs-CRP $\bullet \mathrm{CHFrEF} \bullet \mathrm{CHFpEF}$

\section{Abbreviations}

CHF, chronic heart failure; EF, ejection fraction; CHFrEF, CHF with reduced EF, CHFpEF, CHF with preserved EF; IL1RL1, interleukin 1 receptor like 1; LAVI, left atrial volume index; LVMI, left ventricular mass index; LVEF, left ventricular EF; TAPSE, tricuspid annular plane systolic excursion; 6MWT, the 6-minute walking test; E, transmitral early peak velocity; $\boldsymbol{e}$, early diastolic mitral annulus velocity; NT-proBNP, N-terminal pro-brain natriuretic peptide; hs-CRP, high-sensitivity C-reactive protein.

\section{Introduction}

Chronic heart failure (CHF) is a rapidly growing public health issue associated with increased morbidity and mortality. Modern understanding of the CHF pathogenesis indicates the heterogeneity of the processes occurring in the myocardium, depending on LVEF. ${ }^{(1-3)}$ In the literature, CHFrEF is discussed

*Corresponding author: Evgeniy S. Ovsyannikov, PhD. Department of faculty therapy, Voronezh State Medical University named after N.N. Burdenko. Voronezh, Russia. E-mail: ovses@ yandex.ru in detail. ${ }^{(4)}$ Nevertheless, about half of patients with CHF have LVEF $>50 \%$, which corresponds to CHFpEF, and morbidity and mortality rates are comparable for both groups. ${ }^{(5)}$

It is believed that endogenous low-level inflammation, cellular hypertrophy, and an increase in myocardial stiffness (due to extracellular reorganization) are the determining processes in the formation of CHFpEF. Essentially, CHFpFV is a process of proliferation of connective tissue and disorders of cell metabolism. ${ }^{(6)}$ Various pathogenetic cascades imply the likelihood that various biologically active substances have participated in their implementation. Many biomarkers are being actively studied for their use as methods for diagnosing 
and monitoring the stability of CHF patients. ${ }^{(7)}$ In particular, it is NT-proBNP, hs-CRP, and the less studied ST2 protein.

The Il1rl1 gene product, also having the alias ST2, is a member of the interleukin 1 receptor superfamily. ${ }^{(8,9)}$ For the first time, it has become known as a participant in inflammatory and autoimmune reactions. ${ }^{(10)} \mathrm{ST} 2$ is defined as the IL-33 receptor, as it binds to IL-33. ${ }^{(11,12)} \mathrm{ST} 2$ has two main isoforms: transmembrane or cellular (ST2L) and soluble or circulating (sST2) forms. ${ }^{(13,14)}$ In experimental models, the interaction between IL-33 and ST2L provided the cardioprotective effects, reducing myocardial fibrosis, cardiomyocyte hypertrophy, and apoptosis, as well as improving myocardial function. sST2 avidly binds to IL-33, competing with ST2L. ${ }^{(14)}$ The interaction of sSH2 with IL-33 blocks the IL-33/ST2L system and, as a result, eliminates the cardioprotective effects. SST2 is considered a decoy receptor. ${ }^{(11)}$ Ultimately, a high level of sST2 in blood plasma leads to myocardial fibrosis and dysfunction of the ventricles of the heart. ${ }^{(15)}$ The main sources of sST2 are cardiac fibroblasts and cardiomyocytes in response to stress or injury. Recent studies have demonstrated soluble ST2 to be a strong predictor of cardiovascular outcomes in both chronic and acute heart failure. ${ }^{(14)}$ Consistent with the ADHF data, soluble ST2 has been proven to be useful as a prognostic marker in chronic HF. ${ }^{(16)}$

The weaknesses of the NT-proBNP diagnostic capabilities, namely that its representativeness depends on some comorbid conditions of patients (impaired renal function, anemia, COPD, obesity), encourages scientists to search for a more stable diagnostic marker for patients with a different EF of CHF. Despite the fact that separate studies have already been conducted ${ }^{(7,15,17)}$ to assess the relationship of SST2 with other characteristics of CHF patients with different LVEF, the results obtained are contradictory.

The purpose of this study was to determine the blood concentration of SST2 and evaluate the correlations between sST2 and the clinical, instrumental, and echocardiographic characteristics of patients with $\mathrm{CHFrEF}$ and $\mathrm{CHFpEF}$, as well as to evaluate the sST2 informativeness in comparison with NT-proBNP.

\section{Materials and Methods}

The study involved 160 patients diagnosed with CHF (86 men and 74 women, mean age of $72.4 \pm 8.7$ years), included in the regional register of CHF patients in the Voronezh region. All subjects gave informed consent to participate in the study. According to the LVEF value, the patients were divided into two groups: $\mathrm{CHFpEF}(\mathrm{EF} \geq 50 \%)$ was recorded in 69 people, CHFrEF $(\mathrm{EF}<50 \%)$ - in 91 people. The diagnosis of $\mathrm{CHF}$ was established according to 2016 ESC Guidelines for the diagnosis and treatment of acute and chronic heart failure. ${ }^{(1)}$ The functional class (FC) of CHF was determined according to the NYHA classification (1994), based on the results of the 6MWT. The non-inclusion criteria were the presence of bronchopulmonary diseases(including asthma, COPD), chronic kidney disease( $3 \mathrm{~b}$ stages and higher), diabetes mellitus or taking hypoglycemic drugs, permanent atrial fibrillation, anemia, diseases of musculoskeletal system (coxarthrosis, gonarthrosis, etc., reducing motor activity), obesity (2-3 classes) and other severe somatic pathologies.

Eight weeks after inclusion in the study (patients were examined weekly by the investigator-cardiologist to monitor the stability of the state/course of CHF), participants underwent a standard examination, which included clinical, laboratory and instrumental research methods. For the purpose of an objective assessment of exercise tolerance, a complex of cardiorespiratory analysis was used, designed for functional medical tests, which allows dynamic determination of blood oxygen saturation, heart rate, respiratory rate and the distance in 6MWT (6MWT-D).

The serum levels of NT-proBNP, sST2, and hs-CRP were determined using an automatic analyzer IMMULITE 2000 (Siemens Diagnostics, USA) and quantitative ELISA kits: NT-proBNP - using the Biomedica human NT-proBNP Sandwich ELISA kit (Austria), sST2 - using the Presage ST2 assay kit (Critical Diagnostics, USA), and hs-CRP - using the SRB-IFA-BEST highly sensitive kit (Vector-Best, Russia).

Comprehensive two-dimensional and Doppler echocardiography were performed using an EPIQ5 ultrasound system (Phillips, USA) equipped with S5-1 PureWave Cardiac Transducer. LV end-systolic and end-diastolic volume and left ventricular ejection fraction (LVEF) were calculated by the biplane Simpson method. ${ }^{(18)}$ Early transmitral inflow velocity (E) and early diastolic mitral annular velocity (e') were determined, and an E/e' ratio was acquired. LAVI and TAPSE were also evaluated.

All data was evaluated with STATGRAPHICS Plus 5.1 Median (Me) and interquartile range (IQR; 25th to 75th percentiles) were calculated. Mann-Whitney U test was used for group comparisons. Group comparisons with respect to categorical variables were performed using chi-square tests or, alternatively, Fisher's exact test when expected cell counts were less than 5. Spearman's correlations were used to assess the relationship between NT-proBNP, sST2, hs-CRP levels with echo parameters. A probability value of $\mathrm{P}<0.05$ was considered statistically significant.

\section{Results and Discussion}

The median of NT-proBNP level for patients with CHFrEF was 1804(608-4908) ng/L, which significantly exceeded its value in patients with CHFpEF - 980(301-2677) $\mathrm{ng} / \mathrm{L}(P<0.001)$. The sST2 level was also higher in patients with CHFrEF and amounted to 37(21-56) ng/ml, while for the group of patients with CHFpEF this indicator was 21(15-35) $\mathrm{ng} / \mathrm{ml}(P<0.001)$. sST2 levels in both groups increased with increasing NYHA FC.

In the HFpEF group, the average levels of LAVI and LVMI were 43(37-53) $\mathrm{ml} / \mathrm{m}^{2}$ and $115(95-143) \mathrm{g} / \mathrm{m}^{2}$, respectively. In the HFrEF group, the average levels of LAVI and LVMI were $48(40-73) \mathrm{ml} / \mathrm{m}^{2}$ and $146(127-184) \mathrm{g} / \mathrm{m}^{2}$, respectively. A higher level of sST2 in patients with $\mathrm{HFrEF}$, in combination with more pronounced hypertrophy and myocardial remodeling as described above, indicates that the induction of sST2 is caused by the mechanical overload of cardiomyocytes. Consequently, an increase in the level of this 
biomarker may reflect myocardial stress, the architectonics changes in the heart during the development of CHF and fibrosis.

With regard to the level of hs-CRP, as a biomarker of endogenous inflammatory processes, its value was 3.4(1.28.1) $\mathrm{mg} / \mathrm{L}$ in patients with CHFpEF, while in patients with CHFrEF it was statistically significantly less - 2.9(1.6-5.4) $\mathrm{mg} / \mathrm{L}(P<0.001)$.

The correlation analysis of the sST2 levels and other variables in the study groups produced the following results: NT-proBNP and hs-CRP levels had a stronger positive association with sST2 levels in CHFrEF patients $(\mathrm{r}=0.53$, $P<0.001$ and $\mathrm{r}=0.48, P<0.001$, respectively) than in CHFpEF patients ( $\mathrm{r}=0.40, P<0.001$ and $\mathrm{r}=0.29, P<0.001$, respectively). This is consistent with the theory of heterogeneity of pathological processes in the myocardium in patients with different $\mathrm{EF}^{(1)}$

We found a weak correlation between SST2 and NYHA FC in both groups, and the strength of the correlation slightly increased with an increase in the severity of CHF: FC II ( $\mathrm{r}=0.23$ $[P=0.007]$ in CHFrEF; $\mathrm{r}=0.21[P=0.003]$ in CHFpEF); FC III $(\mathrm{r}=0.25[P=0.004]$ in CHFrEF; $\mathrm{r}=0.24[P<0.001]$ in $\mathrm{CHFpEF})$; FC IV $(\mathrm{r}=0.28[P<0.001]$ in CHFrEF; $\mathrm{r}=0.26[P=0.006]$ in CHFpEF).

The results obtained (regardless of the EF) indicated a statistically significant direct correlation between the levels of sST2 and LAVI $(\mathrm{r}=0.45[P<0.001]$ in CHFpEF; $\mathrm{r}=0.37$ $[P<0.001]$ in $\mathrm{CHFrEF})$, the dysfunction of which can be a marker of the severity of $\mathrm{CHF}^{(19)}$

The study did not reveal a relationship between SST2 levels and LV filling pressure, calculated as E/e' $(\mathrm{r}=0.09$ $[P=0.06]$ in CHFpEF; $\mathrm{r}=0.11[P=0.07]$ in CHFrEF) or LVMI $(\mathrm{r}=0.16[P<0.043]$ in CHFpEF; $\mathrm{r}=0.08[P=0.6]$ in $\mathrm{CHFrEF})$.
In the group of patients with CHFpEF, an inverse correlation was observed between the sST2 level and 6MWT-D ( $\mathrm{r}=-0.69, P=0.007)$ and LVEF $(\mathrm{r}=-0.26, P<0.001)$. In the group of patients with CHFrEF, the level of sST2 also showed an inverse correlation with LVEF $(\mathrm{r}=-0.43, P<0.001)$ and 6MWT-D ( $\mathrm{r}=-0.52, P=0.008)$ (Table 1).

Correlation analysis of the sST2 and NT-proBNP levels with functional, laboratory, instrumental parameters of patients with CHFpEF and CHFrEF revealed the strengths and weaknesses of both biomarkers. The study found a moderate correlation between sST2 levels and LAVI. In turn, LAVI is the most representative echo indicator for detecting $\mathrm{CHF}$ in patients with preserved $\mathrm{EF}$, reflecting the prognosis of the disease.

NT-proBNP has been shown to be an effective biomarker not only for diagnosis but also for prognostic evaluation both for patients with CHFrEF and CHFpEF. ${ }^{(10,20-25)}$ The increased NT-proBNP levels in CHFrEF and HFpEF should be taken as a marker for increased risk of adverse outcomes. However, the predominance of NT-proBNP, as well as sST2 values in patients with $\mathrm{CHFrEF}$, suggests a greater severity of the myocardial remodeling in CHFrEF, confirmed by the results of echocardiography. The data obtained in this study are consistent with the data of other authors on the increased production of NT-proBNP and SST2 in mechanically overloaded cardiomyocytes, which reflects myocardial stress, ventricular remodeling and fibrosis. ${ }^{(26)}$

For 6MWT-D and LVEF, moderate correlations with both biomarkers were also statistically significant. Therefore, the sST2 level, reflecting exercise tolerance and myocardial contractility, can be used as a diagnostic and prognostic marker, like LVEF, especially in situations when it is impossible to perform functional tests and instrumental studies.

Table 1.

Correlations between sST2 and other variables in patients with $\mathrm{CHFpEF}$ and $\mathrm{CHFrEF}$

\begin{tabular}{|c|c|c|c|c|c|c|c|c|}
\hline \multirow{3}{*}{ Variables } & \multicolumn{4}{|c|}{ sST2 } & \multicolumn{4}{|c|}{ NT-proBNP } \\
\hline & \multicolumn{2}{|c|}{ CHFpEF } & \multicolumn{2}{|c|}{ CHFrEF } & \multicolumn{2}{|c|}{ CHFpEF } & \multicolumn{2}{|c|}{ CHFrEF } \\
\hline & $\mathrm{r}$ & $P$ & $\mathrm{r}$ & $P$ & $\mathrm{r}$ & $P$ & $\mathrm{r}$ & $P$ \\
\hline NT-proBNP & 0.53 & $<0.001$ & 0.40 & $<0.001$ & & & & \\
\hline hs-CRP & 0.48 & $<0.001$ & 0.29 & $<0.001$ & 0.29 & 0.007 & 0.19 & 0.009 \\
\hline NYHA FC II & 0.23 & 0.007 & 0.21 & 0.003 & 0.22 & $<0.001$ & 0.32 & 0.005 \\
\hline NYHA FC III & 0.25 & 0.004 & 0.24 & $<0.001$ & 0.26 & 0.005 & 0.39 & $<0.001$ \\
\hline NYHA FC IV & 0.28 & $<0.001$ & 0.26 & 0.006 & 0.29 & $<0.001$ & 0.43 & $<0.001$ \\
\hline $\mathrm{E} / \mathrm{e}^{\prime}$ & 0.09 & 0.06 & 0.11 & 0.07 & 0.22 & 0.043 & 0.17 & 0.020 \\
\hline LAVI & 0.45 & $<0.001$ & 0.37 & $<0.001$ & 0.32 & 0.008 & 0.07 & 0.564 \\
\hline LVMI & 0.16 & 0.043 & 0.08 & 0.6 & 0.13 & $<0.001$ & 0.28 & $<0.001$ \\
\hline LVEF & -0.26 & $<0.001$ & -0.43 & $<0.001$ & -0.17 & $<0.001$ & 0.28 & 0.046 \\
\hline TAPSE & -0.62 & 0.002 & -0.45 & 0.004 & -0.36 & $<0.001$ & -0.24 & $<0.001$ \\
\hline 6MWT D & -0.69 & 0.007 & -0.52 & 0.008 & -0.44 & 0.003 & -0.49 & $<0.001$ \\
\hline
\end{tabular}


Stronger correlations between sST2 and NT-proBNP with hs-CRP in CHFpEF (as compared to CHFrEF) indicate the importance of the contribution of subclinical inflammation to the formation of fibrosis, myocardial remodeling, and, consequently, to the course of CHF. Further accumulation of facts indicating the role of inflammatory processes in the development of CHF may lead to a change in therapeutic approaches in the management of patients with preserved and reduced LVEF.

This study has not revealed a statistically significant correlation between sST2 levels and LV filling pressure. These results are consistent with current research, which also demonstrated the absence of an association between ST2 levels and parameters of LV diastolic function or LV geometry measured by echocardiography. ${ }^{(27)}$

The weak direct correlation between SST2 and NYHA $\mathrm{FC}$, in contrast to the direct strong correlation between NT-proBNP and sST2, indicates that sST2, which is quite important both for determining the current state of the patient and for predicting the course of the disease, still cannot be used as a single and universal indicator. In this regard, further comprehensive study of the diagnostic and prognostic capabilities of the sST2 and NT-proBNP biomarkers in CHF patients with different $\mathrm{EF}$ is required.

In conclusion, the success of using NT-proBNP both in practical medicine (as a reference for the diagnosis of CHF) and in scientific research is limited by many factors that can affect its level. Active studies in this direction have made it possible to identify biomarkers that can deliver additional diagnostic and prognostic information about the patient's condition. Additional information about the likelihood of adverse outcomes can be obtained from the determination of hs-CRP. Thus, its increase (simultaneously with an increase in NT-proBNP) indicates an inflammatory process in the myocardium, which reflects myocardial remodeling. Moreover, it differs from changes in the heart during its hypertrophy and ischemia.

The analysis of SST2 levels allows us to conclude that the prospects are promising for its implementation in the diagnosis and prognosis of CHF since it is a marker of myocardial stress and ventricular remodeling and fibrosis.

\section{Competing Interests}

The authors declare that they have no competing interests.

\section{References}

1. Ponikowski P, Voors AA, Anker SD, Bueno H, Cleland JG, Coats AJ, et al.; Authors/Task Force Members; Document Reviewers. 2016 ESC Guidelines for the diagnosis and treatment of acute and chronic heart failure: The Task Force for the diagnosis and treatment of acute and chronic heart failure of the European Society of Cardiology (ESC). Developed with the special contribution of the Heart Failure Association (HFA) of the ESC. Eur J Heart Fail. 2016 Aug;18(8):891-975. doi: 10.1002/ejhf.592.

2. Budnevsky AV, Shurupova AD, Kravchenko AY, Tokmachev RE. Clinical efficacy of acute respiratory viral infections prevention in patients with chronic heart failure. Ter Arkh. 2019 Mar 30;91(3):36-41. doi: 10.26442/00403660.201 9.03.000111. [Article in Russian].

3. Tokmachev RE, Kravchenko AYa, Budnevsky AV, et al. Activation of pro-inflammatory cytokines in patients with chronic heart failure comorbid with metabolic syndrome. Kardiovaskulyarnaya Trapiya i Profilaktika. 2015;14(2):116117. [Article in Russian].

4. Burnett H, Earley A, Voors AA, Senni M, McMurray JJ, Deschaseaux C, Cope S. Thirty Years of Evidence on the Efficacy of Drug Treatments for Chronic Heart Failure With Reduced Ejection Fraction: A Network Meta-Analysis. Circ Heart Fail. 2017 Jan;10(1):e003529. doi: 10.1161/ CIRCHEARTFAILURE.116.003529.

5. Tschöpe C, Birner C, Böhm M, Bruder O, Frantz S, Luchner A, et al. Heart failure with preserved ejection fraction: current management and future strategies : Expert opinion on the behalf of the Nucleus of the "Heart Failure Working Group" of the German Society of Cardiology (DKG). Clin Res Cardiol. 2018 Jan;107(1):1-19. doi: 10.1007/s00392-017$1170-6$.

6. Chen YT, Wong LL, Liew OW, Richards AM. Heart Failure with Reduced Ejection Fraction (HFrEF) and Preserved Ejection Fraction (HFpEF): The Diagnostic Value of Circulating MicroRNAs. Cells. 2019 Dec 16;8(12):1651. doi: $10.3390 /$ cells8121651.

7. Bielecka-Dabrowa A, Gluba-Brzózka A, MichalskaKasiczak M, Misztal M, Rysz J, Banach M. The multibiomarker approach for heart failure in patients with hypertension. Int J Mol Sci. 2015 May 12;16(5):10715-33. doi: 10.3390/ijms160510715

8. Werenskiold AK, Hoffmann S, Klemenz R. Induction of a mitogen-responsive gene after expression of the Haras oncogene in NIH 3T3 fibroblasts. Mol Cell Biol. 1989 Nov;9(11):5207-14. doi: $10.1128 / \mathrm{mcb} .9 .11 .5207$.

9. Tominaga S. A putative protein of a growth specific cDNA from BALB/c-3T3 cells is highly similar to the extracellular portion of mouse interleukin 1 receptor. FEBS Lett. 1989 Dec 4;258(2):301-4. doi: 10.1016/0014-5793(89)81679-5.

10. Andreasová T, Vránová J, Vondráková D, Sedláčková L, ZákostelskáZJ, Neužil P, Málek F. Role of biomarkers of cardiac remodeling, myofibrosis, and inflammation in assessment of disease severity in euvolemic patients with chronic stable heart failure. J Int Med Res. 2020 Aug;48(8):300060520947869. doi: $10.1177 / 0300060520947869$.

11. Schmitz J, Owyang A, Oldham E, Song Y, Murphy E, McClanahan TK, et al. IL-33, an interleukin-1-like cytokine that signals via the IL-1 receptor-related protein ST2 and induces T helper type 2-associated cytokines. Immunity. 2005 Nov;23(5):479-90. doi: 10.1016/j.immuni.2005.09.015.

12. Liew FY, Girard JP, Turnquist HR. Interleukin-33 in health and disease. Nat Rev Immunol. 2016 Nov;16(11):676689. doi: 10.1038/nri.2016.95.

13. Pascual-Figal DA, Januzzi JL. The biology of ST2: the International ST2 Consensus Panel. Am J Cardiol. 2015 Apr 2;115(7 Suppl):3B-7B. doi: 10.1016/j.amjcard.2015.01.034.

14. Villacorta H, Maisel AS. Soluble ST2 Testing: A Promising Biomarker in the Management of Heart Failure. Arq Bras Cardiol. 2016 Feb;106(2):145-52. doi: 10.5935/ abc. 20150151 .

15. Kuster N, Huet F, Dupuy AM, Akodad M, Battistella P, Agullo A, et al. Multimarker approach including CRP, sST2 and GDF-15 for prognostic stratification in stable heart failure. ESC 
Heart Fail. 2020 Jul 10;7(5):2230-9. doi: 10.1002/ehf2.12680. 16. Bayes-Genis A, Zhang Y, Ky B. ST2 and patient prognosis in chronic heart failure. Am J Cardiol. 2015 Apr 2;115(7 Suppl):64B-9B. doi: 10.1016/j.amjcard.2015.01.043.

17. Tromp J, Westenbrink BD, Ouwerkerk W, van Veldhuisen DJ, Samani NJ, Ponikowski P, et al. Identifying Pathophysiological Mechanisms in Heart Failure With Reduced Versus Preserved Ejection Fraction. J Am Coll Cardiol. 2018 Sep 4;72(10):1081-1090. doi: 10.1016/j. jacc.2018.06.050.

18. Schiller NB, Shah PM, Crawford M, DeMaria A, Devereux $\mathrm{R}$, Feigenbaum $\mathrm{H}$, et al.. Recommendations for quantitation of the left ventricle by two-dimensional echocardiography. American Society of Echocardiography Committee on Standards, Subcommittee on Quantitation of TwoDimensional Echocardiograms. J Am Soc Echocardiogr. 1989 Sep-Oct;2(5):358-67. doi: 10.1016/s0894-7317(89)80014-8. 19. Santos AB, Kraigher-Krainer E, Gupta DK, Claggett B, Zile MR, Pieske B, et al.; PARAMOUNT Investigators. Impaired left atrial function in heart failure with preserved ejection fraction. Eur J Heart Fail. 2014 Oct;16(10):1096-103. doi: 10.1002/ejhf.147.

20. Hartmann F, Packer M, Coats AJ, Fowler MB, Krum H, Mohacsi P, et al. Prognostic impact of plasma N-terminal pro-brain natriuretic peptide in severe chronic congestive heart failure: a substudy of the Carvedilol Prospective Randomized Cumulative Survival (COPERNICUS) trial. Circulation. 2004 Sep 28;110(13):1780-6. doi: 10.1161/01. CIR.0000143059.68996.A7.

21. Olsson LG, Swedberg K, Cleland JG, Spark PA, Komajda M, Metra M, et al.; COMET Investigators. Prognostic importance of plasma NT-pro BNP in chronic heart failure in patients treated with a beta-blocker: results from the Carvedilol Or Metoprolol European Trial (COMET) trial. Eur J Heart Fail. 2007 Aug;9(8):795-801. doi: 10.1016/j. ejheart.2007.07.010.
22. Cleland JG, McMurray JJ, Kjekshus J, Cornel JH, Dunselman P, Fonseca C, et al.; CORONA Study Group. Plasma concentration of amino-terminal pro-brain natriuretic peptide in chronic heart failure: prediction of cardiovascular events and interaction with the effects of rosuvastatin: a report from CORONA (Controlled Rosuvastatin Multinational Trial in Heart Failure). J Am Coll Cardiol. 2009 Nov 10;54(20):1850-9. doi: 10.1016/j.jacc.2009.06.041.

23. Valle R, Aspromonte N, Feola M, Milli M, Canali C, Giovinazzo P, et al. B-type natriuretic peptide can predict the medium-term risk in patients with acute heart failure and preserved systolic function. J Card Fail. 2005 Sep;11(7):498503. doi: 10.1016/j.cardfail.2005.05.002.

24. Anand IS, Rector TS, Cleland JG, Kuskowski M, McKelvie RS, Persson H, et al. Prognostic value of baseline plasma amino-terminal pro-brain natriuretic peptide and its interactions with irbesartan treatment effects in patients with heart failure and preserved ejection fraction: findings from the I-PRESERVE trial. Circ Heart Fail. 2011 Sep;4(5):569-77. doi: 10.1161/CIRCHEARTFAILURE.111.962654.

25. Yan J, Gong SJ, Li L, Yu HY, Dai HW, Chen J, et al. Combination of B-type natriuretic peptide and minute ventilation/carbon dioxide production slope improves risk stratification in patients with diastolic heart failure. Int J Cardiol. 2013 Jan 20;162(3):193-8. doi: 10.1016/j. ijcard.2011.07.017.

26. Santema BT, Chan MMY, Tromp J, Dokter M, van der Wal HH, Emmens JE, et al. The influence of atrial fibrillation on the levels of NT-proBNP versus GDF-15 in patients with heart failure. Clin Res Cardiol. 2020 Mar;109(3):331-338. doi: 10.1007/s00392-019-01513-y.

27. Xanthakis V, Larson MG, Wollert KC, Aragam J, Cheng S, Ho J, et al. Association of novel biomarkers of cardiovascular stress with left ventricular hypertrophy and dysfunction: implications for screening. J Am Heart Assoc. 2013 Nov 7;2(6):e000399. doi: 10.1161/JAHA.113.000399. 